\title{
Invasão de palmeira australiana no sub-bosque de uma floresta plantada, Viçosa, MG
}

\author{
Lucas Siqueira Cardinelli', Rúbia Santos Fonseca², Sebastião Venâncio Martins ${ }^{1}$ \\ 1 'Universidade Federal de Viçosa, Departamento de Engenharia Florestal, Av. Purdue s/n, CEP 36570-900, Viçosa, MG, Brasil \\ Universidade Federal de Minas Gerais, Instituto de Ciências Agrárias, Av. Universitária, CEP 39404-547, Montes Claros, MG, Brasil
}

\author{
*Autor correspondente: \\ lucasscardinelli@hotmail.com
}

Termos para indexação:

Espécies exóticas invasoras

Regeneração da floresta

Araucaria angustifolia

Index terms:

Invasive alien species

Forest rehabilitation

Araucaria angustifolia

Recebido em 07/03/2016

Aprovado em 19/09/2017

Publicado em 30/09/2017

doi: 10.4336/2017.pfb.37.91.1177

\begin{abstract}
Resumo - O objetivo desse trabalho foi avaliar a regeneração natural e a abundância de espécies exóticas no sub-bosque de um plantio experimental de Araucaria angustifolia, em Viçosa, MG. A amostra correspondeu a 40 parcelas de $2 \mathrm{~m}$ x $2 \mathrm{~m}$, onde mediu-se o diâmetro ao nível do solo (DNS) de todos os indivíduos arbustivo-arbóreos com diâmetro $\leq$ a $5,0 \mathrm{~cm}$ e altura $\geq$ a $0,5 \mathrm{~m}$. Foram calculados os parâmetros fitossociológicos e os índices de diversidade e equabilidade. As espécies foram classificadas quanto à forma de vida, classe sucessional e síndrome de dispersão. Foram amostrados 980 indivíduos, sendo observada maior proporção de espécies arbustivas, típicas de sub-bosque e zoocóricas. A espécie exótica Archontophoenix cunninghamiana, conhecida como palmeira australiana, obteve o terceiro maior valor de importância, a maior dominância e 19,25\% da área basal total, valor consideravelmente maior que o das demais espécies. A. cunninghamiana foi caracterizada como uma espécie exótica invasora que domina o estrato de regeneração natural. A inibição da sucessão natural pela presença de espécies exóticas, como A. cunninghamiana, precisa ser monitorada, visando eliminar as espécies-problema, para garantir a continuidade do processo de sucessão.
\end{abstract}

\section{Invasion of Australian palm in the understory of a planted forest, in Viçosa, MG, Brazil}

\begin{abstract}
This study aimed to evaluate the natural regeneration and the abundance of exotic palms in the understory of a planted forest of Araucaria angustifolia in Viçosa, Minas Gerais State, Brazil. The sample corresponded to 40 plots of $2 \mathrm{~m} \times 2 \mathrm{~m}$. Diameter at ground level of all shrubs and trees with diameter $\leq 5 \mathrm{~cm}$ and height $\geq 0.5$ $\mathrm{m}$ was measured. Phytosociological parameters and diversity indices were calculated. All species were classified regarding its life form, successional class and dispersion syndrome. We sampled 980 individuals that presented higher proportion of shrub species, typical of understory and zoochoric. The exotic species Archontophoenix cunninghamiana, known as Australian palm, presented the third largest importance value, highest dominance and $19.25 \%$ of the total basal area, considerably larger than the other species. A. cunninghamiana was characterized as an invasive species that dominate the natural regeneration stratum. Inibition of natural succession by exotic species, such as A. cunninghamiana, should be monitored, aiming at the withdrawal of the problem species what will ensure the succession process.
\end{abstract}




\section{Introdução}

Povoamentos florestais são tradicionalmente implantados para a exploração econômica de madeira, selecionando espécies de elevado crescimento e produtividade vegetal, o que envolve, ao final de cada ciclo, o desbaste dos indivíduos adultos, a limpeza do seu sub-bosque e o preparo da terra para o início de um novo ciclo de produção (Paiva et al., 2011). No entanto, a capacidade de crescimento dessas espécies pode ser utilizada como ferramenta para a recuperação de comunidades florestais complexas e ecologicamente ativas (Isernhagen et al., 2009). Nesses casos, os indivíduos adultos são mantidos em pé e não é feita retirada do sub-bosque. O sucesso dessa técnica está relacionado à escolha da espécie, em função de características como seu ciclo de vida, potencial alelopático e a sua interação com as espécies típicas das vegetações locais (Callegaro et al., 2013; Melo et al., 2015).

Os plantios florestais, incluindo os monocultivos, podem ser utilizados como estratégia de restauração em ambientes florestais degradados, pois criam rapidamente micro-habitats propícios à colonização do sub-bosque (Callegaro et al., 2013). O sub-bosque, por sua vez, atua como catalisador da regeneração de espécies arbustivoarbóreas nativas. Com o passar do tempo, os indivíduos plantados cedem espaço para a floresta secundária típica (Onofre et al., 2010; Ribeiro et al., 2012; Miranda-Neto et al., 2012; Callegaro et al., 2013). Entretanto, esse processo de enriquecimento e substituição florística pode ser atrasado ou redirecionado quando espécies exóticas passam a colonizar agressivamente o sub-bosque e a monopolizar os recursos, gerando um típico modelo de inibição da sucessão (Connel \& Slatyer, 1977; Denslow \& DeWalt, 2008).

A invasão de espécies exóticas é a segunda maior causa de perda de biodiversidade em todo o mundo, atrás somente da destruição de habitats (Wilcove et al., 1998). O monitoramento de espécies exóticas invasoras tem sido reconhecido como importante indicador do nível de degradação de ecossistemas naturais (Thompson et al., 2013), devido à relação existente entre o aumento da abundância (biomassa, densidade) dessas espécies e a redução de bens e serviços ambientais no ecossistema invadido (Richardson \& Wilgen, 2004).

Por meio de levantamentos florísticos e fitossociológicos é possível detectar o nível da invasão de espécies vegetais exóticas no sub-bosque de uma comunidade florestal (Callegaro et al., 2013). Para tanto, é necessário mensurar a representatividade das populações dessas espécies na organização estrutural da comunidade avaliada. Estudos dessa natureza são essenciais para a definição de estratégias de manejo visando à eliminação das espécies invasoras do sub-bosque de florestas homogêneas plantadas com finalidade de restauração da diversidade vegetal e funcionalidade do ecossistema (Durigan \& Engel, 2012).

O objetivo desse trabalho foi avaliar a estrutura da regeneração natural, a distribuição dos grupos sucessionais e a abundância de espécies exóticas no sub-bosque de uma floresta plantada de Araucaria angustifolia (Bertol.) Kuntze, visando subsidiar ações de manejo que evitem ou reduzam a invasão biológica e contribuam para a regeneração das espécies nativas.

\section{Material e métodos}

O estudo foi realizado na Reserva da Biologia, situada no campus da Universidade Federal de Viçosa (UFV), Viçosa, MG. O clima da região é do tipo Cwa (segundo classificação de Köppen), subtropical úmido com invernos secos e verões quentes, apresentando $21,8{ }^{\circ} \mathrm{C}$ de temperatura média anual, $1.361 \mathrm{~mm}$ de precipitação média anual (Alvares et al., 2013) e $650 \mathrm{~m}$ de altitude. O município de Viçosa está imerso no contínuo da Floresta Estacional Semidecidual do Domínio Atlântico (Oliveira-Filho \& Fontes, 2000).

A Reserva da Biologia possui 75 ha, que estão protegidos de desmatamento há 90 anos (Ferreira-Junior et al., 2007). Essa reserva tem as funções de conservar a biodiversidade da Floresta Estacional Semidecidual e permitir a execução de pesquisas em comunidades florestais (Ferreira-Junior et al., 2007; Camargos et al., 2010). Na área de borda desse fragmento, segundo a Divisão de Parques e Jardins da UFV, há 40 anos foi realizado o plantio experimental de Araucaria angustifolia, que desde então foi protegido e mantido sem ações de manejo. O reduzido nível de interferência humana e a proximidade com áreas de floresta preservada permitiu a formação de um dossel contínuo, com cerca de $15 \mathrm{~m}$ de altura. No sub-bosque desse plantio observa-se a regeneração de espécies arbustivo-arbóreas, típicas de Floresta Estacional Semidecidual da região. Entretanto, nessa comunidade são observadas espécies exóticas, como as palmeiras Archontophoenix cunninghamiana 
H. Wendl. \& Drude (palmeira-australiana) e Livistona chinensis (Jacq.) R. Br. ex Mart. (palmeira-leque), que foram introduzidas, provavelmente, pela dispersão de sementes provenientes dos indivíduos adultos plantados no campus da UFV (Eisenlohr et al., 2008). Os frutos dessas espécies são consumidos pela avifauna local, sobretudo pelo jacú (Penelope obscura), ave comum na área de estudo (observação pessoal). Essa ave libera boa parte das sementes intactas após passagem dos frutos pelo seu trato digestivo (Christianini, 2006), atuando como dispersor.

Araucaria angustifolia é uma árvore heliófita, característica de regiões de clima subtropical (Carvalho, 1994), sendo o componente dominante da Floresta Ombrófila Mista (IBGE, 2012). Essa espécie ocorre naturalmente em áreas do sudeste e sul do Brasil (Reflora, 2016). No Sudeste, pode ser encontrada em Minas Gerais, Rio de Janeiro e São Paulo, em regiões de altitude acima de 900 m (Carvalho, 1994; Reflora, 2016). A. angustifolia não é nativa de Viçosa, sendo introduzida nesse município (Eisenlohr et al., 2008).

$\mathrm{Na}$ área de estudo, foram alocadas ao acaso 40 parcelas de $2 \mathrm{~m} \times 2 \mathrm{~m}$. Em cada parcela foi mensurado o diâmetro ao nível do solo (DNS) de todos os indivíduos arbustivo-arbóreos com DNS $\leq 5,0 \mathrm{~cm}$ e altura $\geq$ a $0,5 \mathrm{~m}$. Plantas que emergiram separadamente ao nível do solo foram consideradas indivíduos distintos sensu Moro \& Martins (2011).

A classificação botânica das espécies seguiu o APG IV (The Angiosperm Phylogeny Group, 2016). Os nomes científicos e a classificação quanto à origem (nativa ou exótica) seguiu a Lista de Espécies da Flora do Brasil (Reflora, 2016).

As espécies também foram classificadas quanto à forma de vida em árvore, arbusto ou palmeira (Font Quer \& Sierra Ràfols, 1979), quanto à categoria sucessional em pioneira, secundária inicial, secundária tardia ou típica de sub-bosque (completam todo ciclo de vida no sub-bosque) (Gandolfi et al., 1995) e quanto à síndrome de dispersão em autocórica, anemocórica ou zoocórica sensu Pijl (1982). Para a definição das síndromes de dispersão, utilizou-se sementes e frutos coletados na área, além de material de herbário. Os diásporos foram avaliados quanto ao tamanho, cor, odor, morfologia e estruturas atrativas aos dispersores.

Foram calculados os parâmetros fitossociológicos de densidade, frequência e dominância absolutas e relativas, valor de importância (Müller-Dombois \& Ellemberg,
1974), índice de diversidade de Shannon-Wiener (H') (Magurran, 2011) e equabilidade de Pielou (J') (Pielou, 1975).

\section{Resultados e discussão}

A comunidade avaliada apresentou 980 indivíduos, correspondendo a 61.250 indivíduos ha ${ }^{-1}$, que somaram $0,083 \mathrm{~m}^{2}$ de área basal $\left(5,195 \mathrm{~m}^{2} \mathrm{ha}^{-1}\right)$. A densidade observada foi superior a de outros trabalhos realizados na região de Viçosa, MG (Silva Júnior et al., 2004; Marangon et al., 2008; Miranda-Neto et al., 2012). Essa diferença pode ser atribuída, principalmente, à elevada densidade das espécies de Piperaceae.

Os indivíduos avaliados pertencem a 64 espécies e 22 famílias botânicas. Dessas, 50 foram identificadas até o nível de espécie, seis a gênero, três a família e cinco permaneceram indeterminadas. A riqueza de espécies foi inferior a outros levantamentos de regeneração na região de Viçosa, MG (Marangon et al., 2008; Miranda-Neto et al., 2012). Miranda Neto et al. (2012), avaliando a regeneração natural no subbosque de um reflorestamento heterogêneo, em Viçosa, MG, encontraram elevado número de espécies (103). Entretanto, apesar da maior riqueza dessa floresta, também observaram maior número de espécies exóticas (7), quando comparado ao presente estudo (3), resultado da composição de espécies plantadas ( $25 \%$ das espécies plantadas não eram nativas do Brasil, incluindo as sete espécies exóticas regenerantes). Dentre essas espécies exóticas, os autores deram destaque a Archontophoenix cunninghamiana, recrutada em $62 \%$ da área amostral, apesar de originalmente terem sido plantados apenas cinco indivíduos dessa espécie. A escolha das espécies a serem plantadas deve levar em conta a capacidade dessas de invadirem ambientes naturais e formarem comunidades dominantes.

Apesar de não ser nativa da Floresta Estacional Semidecidual, o plantio monodominante de Araucaria angustifolia proporcionou ambiente adequado para a regeneração natural de plantas autóctones, atuando como facilitador. Nesse contexto, a facilitação, que é um modelo de sucessão no qual as últimas espécies só são capazes de se estabelecerem após as primeiras terem modificado o ambiente adequadamente (Connell \& Slatyer, 1977), depende muito mais da capacidade de modificar o ambiente do que da origem da espécie facilitadora (Melo et al., 2015). 
Dentre os atributos morfológicos da Araucaria angustifolia associados ao seu funcionamento como facilitadora da regeneração natural, destacam-se o porte elevado e a largura e arquitetura da copa. Árvores com copa alta e larga podem funcionar como poleiros ou posto de observação de pássaros e outros animais, sendo priorizadas na seleção de espécies atrativas à fauna dispersora de sementes (Melo et al., 2015).

Por outro lado, o crescimento lento da araucária (Beckert et al., 2014) pode ser uma característica problemática para indicá-la em projetos de recuperação de área degradada, em que a produtividade e taxa de crescimento das espécies são cruciais. Entretanto, como componente de projeto paisagístico envolvendo a conservação de habitats para espécies nativas, como é o caso do presente estudo, o crescimento lento da araucária não é prejudicial. Dessa forma, $A$. angustifolia pode ser indicada como árvore ornamental em paisagens com cobertura florestal de Mata Atlântica, mesmo fora da Floresta Ombrófila Mista, desde que em ambiente com clima adequado para sua sobrevivência (Eisenlohr et al., 2008; Longhi, et al., 2010).

$\mathrm{O}$ índice de diversidade registrado $\left(\mathrm{H}^{\prime}=2,79\right)$ foi inferior aos outros levantamentos da comunidade regenerante em Floresta Estacional Semidecidual madura na região de Viçosa, os quais variaram de 3,15 a 3,6 (Silva Júnior et al., 2004; Marangon et al., 2008; Miranda-Neto et al., 2012). Da mesma forma, o índice de equabilidade de Pielou $\left(\mathrm{J}^{\prime}=0,672\right)$ foi inferior aos das áreas maduras $\left(\mathrm{J}^{\prime}=0,8\right)$. Esses resultados demonstram que a comunidade regenerante do plantio florestal apresenta maior dominância ecológica e é floristicamente mais homogênea que a regeneração dos fragmentos de floresta madura na mesma região (Silva Júnior et al., 2004; Marangon et al., 2008; Miranda-Neto et al., 2012).

A dominância ecológica na área foi exercida, principalmente, por Piper pubisubmarginalum Yunck. e $P$. lucaeanum Kunth (Tabela 1). Em estudo realizado em sub-bosque na Mata da Silvicultura, em Viçosa, $P$. lucaeunum foi a segunda espécie mais relevante e obteve o maior valor de importância (VI) (MeiraNeto \& Martins, 2003), o que indica que as condições ecológicas para tal espécie foram adequadas em ambas situações. Por outro lado, em estudo de regeneração realizado em área de Floresta Semidecídua em estágio inicial de sucessão observou-se H' $=1,91$ e J' $=0,45$ (Silva Júnior et al., 2004), resultados inferiores aos da área de estudo, demonstrando que o dossel do plantio homogêneo é capaz de manter uma comunidade de subbosque mais complexa que as de florestas no início da sucessão secundária.

Em relação ao número de espécies, os arbustos corresponderam a $39 \%$ da riqueza total, as árvores a $37,5 \%$ e as palmeiras, com duas espécies, a 3,13\% (Figura 1a). A proporção, em número de indivíduos, de arbustos, árvores e palmeiras foi de $79,1 \%, 6,7 \%$ e $14,2 \%$, respectivamente (Figura $1 \mathrm{~b}$ ). A maior densidade de arbustos está associada à elevada densidade das espécies do gênero Piper, todas arbustivas na estrutura do sub-bosque. Por outro lado, a menor densidade de árvores regenerantes demonstra que a resiliência da floresta pode estar comprometida. Um fator crítico na dinâmica de ecossistemas florestais é o incremento de árvores no estrato regenerante. A densidade de árvores nesse estrato representa o potencial de perpetuação das mesmas no ecossistema, pois esses indivíduos vão compor o dossel da floresta, mantendo o processo de sucessão natural. Ademais, a comunidade regenerante contribui com serviços ambientais, como sombreamento do sub-bosque, produção de serapilheira e regulação de temperatura e umidade (Callegaro et al., 2013).

As espécies típicas de sub-bosque (SB) predominaram sobre os demais grupos (pioneiras e secundárias), tanto em número de espécies (Figura 1c), como de indivíduos (Figura 1d). Do total de indivíduos amostrados, 77,7\% foram de espécies que completam todo seu ciclo de vida no sub-bosque, onde se destacaram Aphelandra schottiana (Nees.) Profice, Psychotria rhytidocarpa Müll. Arg. e Piper spp. Espécies do gênero Piper ocorrem preferencialmente em ambientes úmidos e moderadamente sombreados, como bordas de fragmentos de florestas tropicais ou margens de clareiras e trilhas abertas no interior desses fragmentos (Cavalheiro et al., 2013). O grupo de espécies típicas de sub-bosque é representado por plantas de pequeno porte, predominantemente arbustos e arvoretas tolerantes à sombra e, geralmente, dispersas por animais.

O predomínio de espécies tolerantes ao sombreamento no sub-bosque do povoamento é resposta ao dossel contínuo proporcionado pelas árvores de Araucaria angustifolia, criando condições para espécies de subbosque, comuns em estágios avançados de sucessão. Isso pode ocorrer devido à similaridade de condições microclimáticas com o sub-bosque de florestas nativas, já observada em outras áreas de florestas plantadas (Souza et al., 2007; Onofre et al., 2010). 
Tabela 1. Estrutura fitossociológica do estrato de regeneração do sub-bosque de uma floresta plantada com Araucaria angustifolia em Viçosa, MG.

\begin{tabular}{|c|c|c|c|c|c|c|c|c|c|}
\hline Espécie & FV & GS & SD & $\mathbf{O}$ & $\mathbf{N}$ & DA & DoA & FA & VI \\
\hline Piper pubisubmarginalum & Arb. & SB & Zoo. & Nt. & 234 & 14625 & 0,2856 & 45 & 36,09 \\
\hline Piper lucaeanum & Arb. & SB & Zoo. & Nt. & 189 & 11813 & 0,3276 & 50 & 33,05 \\
\hline Archontophoenix cunninghamiana & Palm. & $\mathrm{NC}$ & Zoo. & Ex. & 44 & 2750 & 1,0004 & 45 & 30,46 \\
\hline Aphelandra schottiana & Arb. & SB & Auto. & Nt. & 63 & 3938 & 0,2213 & 52,5 & 18,52 \\
\hline Piper vicosanum & Arb. & $\mathrm{SB}$ & Zoo. & Nt. & 71 & 4438 & 0,1043 & 47,5 & 16,34 \\
\hline Piper gaudichaudianum & Arb. & $\mathrm{SB}$ & Zoo. & Nt. & 22 & 1375 & 0,4456 & 25 & 14,55 \\
\hline Piper crassinervium & Arb. & SB & Zoo. & Nt. & 56 & 3500 & 0,1636 & 30 & 13,34 \\
\hline Ficus adhatodifolia & Arv. & SI & Zoo. & Nt. & 14 & 875 & 0,4426 & 20 & 12,93 \\
\hline Anadenanthera peregrina & Arv. & SI & Anemo. & Nt. & 11 & 687,5 & 0,3537 & 20 & 10,92 \\
\hline Psychotria rhytidocarpa & Arb. & $\mathrm{SB}$ & Zoo. & Nt. & 16 & 1000 & 0,2208 & 17,5 & 8,49 \\
\hline Indeterminado 1 & $\mathrm{NC}$ & $\mathrm{NC}$ & $\mathrm{NC}$ & $\mathrm{NC}$ & 41 & 2563 & 0,1267 & 12,5 & 8,49 \\
\hline Siparuna guianensis & Arb. & SB & Zoo. & Nt. & 9 & 562,5 & 0,2072 & 20 & 7,89 \\
\hline Rudgea sessilis & Arb. & SB & Zoo. & Nt. & 11 & 687,5 & 0,2054 & 17,5 & 7,69 \\
\hline Piper spp. 2 & Arb. & SB & Zoo. & $\mathrm{NC}$ & 46 & 2875 & 0,0211 & 10 & 6,59 \\
\hline Siparuna brasiliensis & Arb. & SB & Zoo. & Nt. & 8 & 500 & 0,1485 & 15 & 5,91 \\
\hline Leandra reversa & Arb. & SI & Zoo. & Nt. & 16 & 1000 & 0,0202 & 22,5 & 5,38 \\
\hline Piper arboreum & Arb. & SB & Zoo. & Nt. & 13 & 812,5 & 0,0156 & 12,5 & 3,49 \\
\hline Ossaea marginata & Arb. & $\mathrm{NC}$ & Zoo. & Nt. & 15 & 937,5 & 0,0165 & 10 & 3,34 \\
\hline Hieronyma alchorneoides & Arv. & SI & Zoo. & Nt. & 2 & 125 & 0,1003 & 5 & 2,88 \\
\hline Casearia decandra & Arv. & SI & Zoo. & Nt. & 5 & 312,5 & 0,0394 & 10 & 2,76 \\
\hline Urera baccifera & Arb. & $\mathrm{P}$ & Zoo. & Nt. & 5 & 312,5 & 0,0115 & 12,5 & 2,60 \\
\hline Guarea guidonia & Arv. & ST & Zoo. & Nt. & 3 & 187,5 & 0,0689 & 5 & 2,38 \\
\hline Psychotria hastisepala & Arb. & SB & Zoo. & Nt. & 7 & 437,5 & 0,0265 & 7,5 & 2,34 \\
\hline Cupania ludowigii & Arv. & SI & Zoo. & Nt. & 4 & 250 & 0,0226 & 10 & 2,34 \\
\hline Copaifera langsdorffii & Arv. & SI & Zoo. & Nt. & 2 & 125 & 0,0634 & 5 & 2,17 \\
\hline Livistona chinensis & Palm. & $\mathrm{NC}$ & Zoo. & Ex. & 1 & 62,5 & 0,0841 & 2,5 & 2,09 \\
\hline Piper caldense & Arb. & SB & Zoo. & Nt. & 5 & 312,5 & 0,0233 & 7,5 & 2,08 \\
\hline Ocotea spp. 3 & $\mathrm{NC}$ & ST & Zoo. & $\mathrm{NC}$ & 7 & 437,5 & 0,0122 & 7,5 & 2,07 \\
\hline Sapium glandulosum & Arv. & $\mathrm{P}$ & Zoo. & Nt. & 1 & 62,5 & 0,0776 & 2,5 & 1,97 \\
\hline Solanum cernuит & Arb. & $\mathrm{P}$ & Zoo. & Nt. & 6 & 375 & 0,0121 & 7,5 & 1,96 \\
\hline Cedrela fissilis & Arv. & SI & Anemo. & Nt. & 3 & 187,5 & 0,0246 & 7,5 & 1,90 \\
\hline Ocotea puberula & Arv. & ST & Zoo. & Nt. & 4 & 250 & 0,0155 & 7,5 & 1,83 \\
\hline Cecropia hololeuca & Arv. & $\mathrm{P}$ & Zoo. & Nt. & 3 & 187,5 & 0,0165 & 7,5 & 1,74 \\
\hline Trichilia pallida & Arv. & ST & Zoo. & Nt. & 2 & 125 & 0,0320 & 5 & 1,57 \\
\hline Alchornea glandulosa & Arv. & $\mathrm{P}$ & Zoo. & Nt. & 2 & 125 & 0,0180 & 5 & 1,30 \\
\hline Ocotea spp. 1 & $\mathrm{NC}$ & ST & Zoo. & $\mathrm{NC}$ & 3 & 187,5 & 0,0105 & 5 & 1,25 \\
\hline Citrus spp. & $\mathrm{NC}$ & $\mathrm{NC}$ & Zoo. & Ex. & 1 & 62,5 & 0,0393 & 2,5 & 1,23 \\
\hline Piper spp. 1 & Arb. & SB & Zoo. & $\mathrm{NC}$ & 4 & 250 & 0,0021 & 5 & 1,19 \\
\hline Cestrum schlechtendalii & Arb. & $\mathrm{P}$ & Zoo. & Nt. & 2 & 125 & 0,0033 & 5 & 1,01 \\
\hline Rubus sellowii & Arb. & SB & Zoo. & Nt. & 2 & 125 & 0,0020 & 5 & 0,99 \\
\hline
\end{tabular}

Continua... 
Tabela 1. continuação.

\begin{tabular}{|c|c|c|c|c|c|c|c|c|c|}
\hline Espécie & FV & GS & SD & $\mathbf{O}$ & $\mathbf{N}$ & DA & DoA & FA & VI \\
\hline Indeterminado 2 & $\mathrm{NC}$ & $\mathrm{NC}$ & $\mathrm{NC}$ & $\mathrm{NC}$ & 1 & 62,5 & 0,0205 & 2,5 & 0,87 \\
\hline Piper mollicomum & Arb. & SB & Zoo. & Nt. & 2 & 125 & 0,0150 & 2,5 & 0,87 \\
\hline Senna affinis & Arv. & $\mathrm{NC}$ & Auto. & Nt. & 2 & 125 & 0,0129 & 2,5 & 0,83 \\
\hline Indeterminado 5 & $\mathrm{NC}$ & $\mathrm{NC}$ & $\mathrm{NC}$ & $\mathrm{NC}$ & 1 & 62,5 & 0,0171 & 2,5 & 0,80 \\
\hline Indeterminado 4 & $\mathrm{NC}$ & $\mathrm{NC}$ & $\mathrm{NC}$ & $\mathrm{NC}$ & 1 & 62,5 & 0,0162 & 2,5 & 0,79 \\
\hline Ocotea spp. 2 & $\mathrm{NC}$ & ST & Zoo. & $\mathrm{NC}$ & 1 & 62,5 & 0,0156 & 2,5 & 0,78 \\
\hline Indeterminado 3 & $\mathrm{NC}$ & $\mathrm{NC}$ & $\mathrm{NC}$ & $\mathrm{NC}$ & 1 & 62,5 & 0,0147 & 2,5 & 0,76 \\
\hline Handroanthus chrysotrichus & Arv. & SI & Anemo. & Nt. & 1 & 62,5 & 0,0140 & 2,5 & 0,75 \\
\hline Ruellia brevifolia & Arb. & SB & Auto. & Nt. & 2 & 125 & 0,0025 & 2,5 & 0,62 \\
\hline Rubiaceae 2 & $\mathrm{NC}$ & $\mathrm{NC}$ & $\mathrm{NC}$ & $\mathrm{NC}$ & 1 & 62,5 & 0,0075 & 2,5 & 0,62 \\
\hline Protium warmingianum & Arv. & $\mathrm{ST}$ & Zoo. & Nt. & 1 & 62,5 & 0,0060 & 2,5 & 0,59 \\
\hline Myrtaceae & $\mathrm{NC}$ & $\mathrm{NC}$ & $\mathrm{NC}$ & $\mathrm{NC}$ & 1 & 62,5 & 0,0039 & 2,5 & 0,55 \\
\hline Eugenia uniflora & Arv. & ST & Zoo. & Nt. & 1 & 62,5 & 0,0036 & 2,5 & 0,55 \\
\hline Allophylus sericeus & Arv. & SI & Zoo. & Nt. & 1 & 62,5 & 0,0027 & 2,5 & 0,53 \\
\hline Machaerium nyctitans & Arv. & SI & Anemo. & Nt. & 1 & 62,5 & 0,0024 & 2,5 & 0,52 \\
\hline Zanthoxylum rhoifolium & Arv. & $\mathrm{P}$ & Zoo. & Nt. & 1 & 62,5 & 0,0020 & 2,5 & 0,51 \\
\hline Piper umbellatum & Arb. & SB & Zoo. & Nt. & 1 & 62,5 & 0,0019 & 2,5 & 0,51 \\
\hline Solanum decompositiflorum & Arb. & $\mathrm{P}$ & Zoo. & Nt. & 1 & 62,5 & 0,0013 & 2,5 & 0,50 \\
\hline Tapirira guianensis & Arv. & SI & Zoo. & Nt. & 1 & 62,5 & 0,0011 & 2,5 & 0,50 \\
\hline Miconia pusilliflora & Arv. & $\mathrm{P}$ & Zoo. & Nt. & 1 & 62,5 & 0,0008 & 2,5 & 0,49 \\
\hline Miconia budlejoides & Arv. & $\mathrm{P}$ & Zoo. & Nt. & 1 & 62,5 & 0,0006 & 2,5 & 0,49 \\
\hline Ocotea dispersa & Arv. & ST & Zoo. & Nt. & 1 & 62,5 & 0,0004 & 2,5 & 0,48 \\
\hline Rubiaceae 1 & $\mathrm{NC}$ & $\mathrm{NC}$ & $\mathrm{NC}$ & $\mathrm{NC}$ & 1 & 62,5 & 0,0002 & 2,5 & 0,48 \\
\hline Myrcia splendens & Arv. & SI & Zoo. & Nt. & 1 & 62,5 & 0,0002 & 2,5 & 0,48 \\
\hline Total geral & & & & & 980 & 61250 & 5,195 & 670 & 300 \\
\hline
\end{tabular}

$\mathrm{FV}=$ forma de vida; $\mathrm{Arv}=$ árvore; $\mathrm{Arb}$ = arbusto, $\mathrm{Palm}=$ palmeira; $\mathrm{NC}$ = não classificada; $\mathrm{GS}$ = grupo sucessional; $\mathrm{SB}$ = típica de sub-bosque; $\mathrm{P}=$ pioneira; $\mathrm{SI}=$ secundária inicial; $\mathrm{ST}$ = secundária tardia; $\mathrm{SD}$ = síndrome de dispersão; Zoo = zoocóricas; Anemo = anemocórica; Auto $=$ autocórica; $\mathrm{O}=$ origem; $\mathrm{Nt}=$ nativa; $\mathrm{Ex}=$ exótica; $\mathrm{DA}=$ densidade absoluta (indivíduos ha $\left.{ }^{-1}\right) ; \mathrm{DoA}=$ dominância absoluta $\left(\mathrm{m}^{2} \mathrm{ha}^{-1}\right) ; \mathrm{FA}=$ frequência absoluta $(\%)$; VI = valor de importância

Entretanto, a baixa proporção deárvores na comunidade regenerante pode comprometer a sustentabilidade da floresta em regeneração. O estudo da dinâmica florestal a partir de levantamentos periódicos na comunidade é uma ferramenta útil para o monitoramento da área, o que permitiria, por exemplo, inferir sobre o avanço ou declínio do número de árvores no dossel em um determinado intervalo de tempo.

O predomínio de indivíduos e espécies zoocóricas sobre as demais síndromes de dispersão no estrato de regeneração do presente trabalho (Figuras 1e e 1f) corrobora com os valores de outros trabalhos em Floresta Estacional Semidecidual, em Viçosa, MG (Miranda Neto et al., 2012). O fluxo de animais é de grande importância para o aumento da biodiversidade local e para a conservação de diversas espécies de plantas nativas, por serem, muitos deles, os responsáveis pela polinização, dispersão e, consequente, recrutamento de plântulas no sub-bosque. Por outro lado, as plantas do sub-bosque propiciam habitat e alimento aos animais, destacandose as espécies de Piper, comumente dispersas por morcegos. A relação mutualística entre fauna e flora explica o sucesso e importância das plantas zoocóricas nos ecossistemas tropicais e na aceleração da sucessão em áreas degradadas (Jordano et al., 2006).

A espécie exótica Archontophoenix cunninghamiana H. Wendl. \& Drude (palmeira-australiana) obteve o terceiro maior VI (Tabela 1) e apresentou a maior dominância, 19,25\% da área basal total. Essas variáveis são indicativas da grande biomassa acumulada por essa 
espécie e da sua capacidade de competição por recursos com as espécies nativas. $\mathrm{O}$ raciocínio utilizado no uso da densidade do invasor como medida do impacto é que qualquer biomassa, espaço ou energia controlada pelo invasor representa recursos não mais disponíveis para as demais espécies da área (Parker et al., 1999).
A

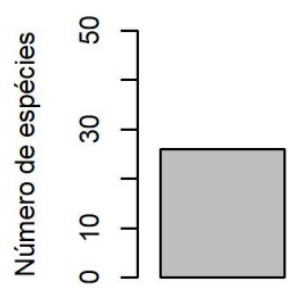

Arb

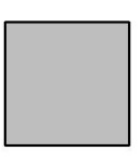

Arv

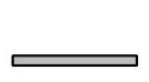

Palm

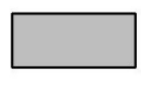

NC

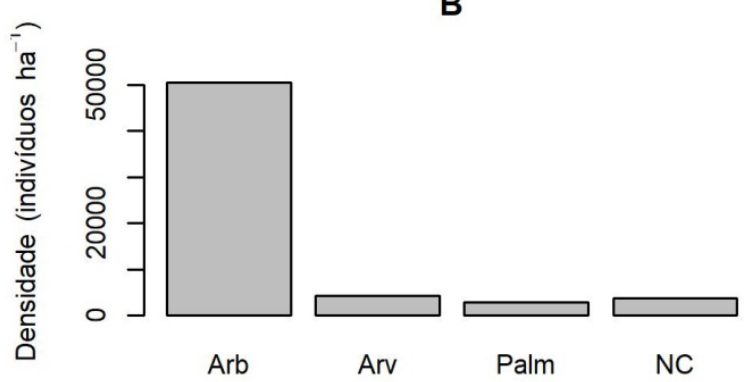

B

D
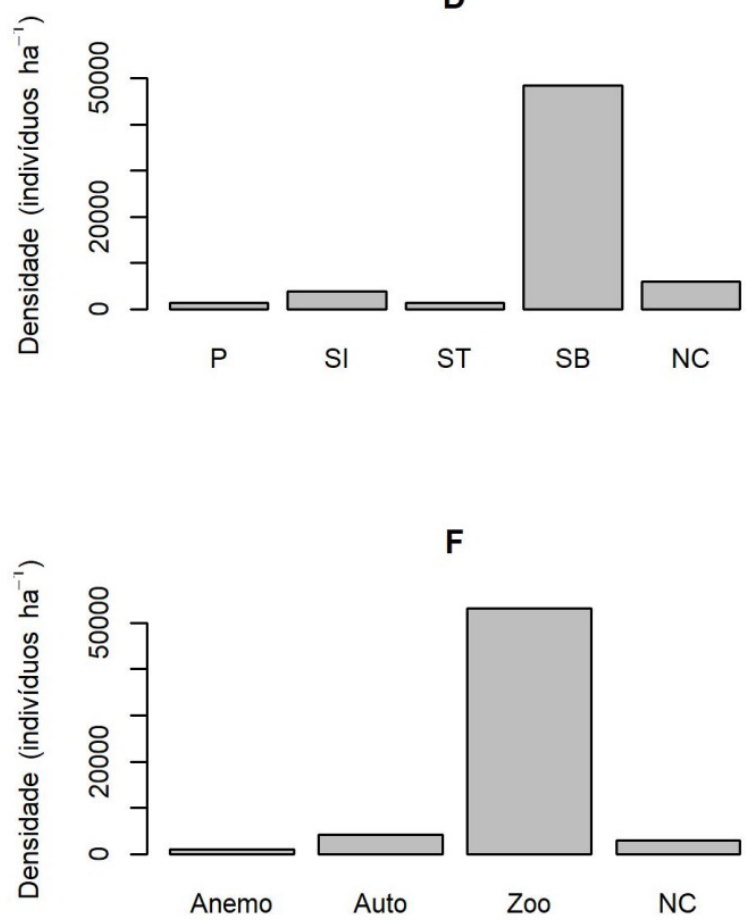

Figura 1. Número de espécies e densidade de indivíduos (indivíduos ha ${ }^{-1}$ ) por forma de vida (A e B), grupo sucessional (C e D) e síndrome de dispersão (E e F) no estrato de regeneração de uma floresta plantada de Araucaria angustifolia, em Viçosa, $\mathrm{MG}$. Arb = arbusto, Arv = árvore, $\mathrm{Palm}=$ palmeira, $\mathrm{NC}$ = não classificada, $\mathrm{P}=$ pioneira, $\mathrm{SI}=$ secundária inicial, $\mathrm{ST}=$ secundária tardia, $\mathrm{SB}=$ sub-bosque, Anemo = anemocórica, Auto = autocórica, $\mathrm{Zoo}=$ zoocórica. 
A invasão da palmeira $A$. cunninghamiana em fragmentos florestais também foi observada no município de São Paulo (Dislich et al., 2002), onde a espécie apresentou o segundo maior VI. Em Viçosa, $A$. cunninghamiana foi relatada como espécie invasora $\mathrm{e}$ assumiu a terceira posição de VI na estrutura do subbosque de uma floresta restaurada com 40 anos (Miranda Neto et al., 2012). A proximidade de fragmentos florestais a áreas urbanas predispõe um contato maior destes remanescentes com espécies exóticas cultivadas, as quais podem potencialmente colonizá-los, como é o caso de $A$. cunninghamiana, nativa da Austrália e comumente utilizada como ornamental no campus da Universidade Federal de Viçosa (Eisenlohr et al., 2008), inclusive em vias adjacentes à floresta plantada de Araucaria angustifolia.

A maioria das espécies exóticas introduzidas que se tornaram invasoras são heliófitas e intolerantes ao sombreamento e, dessa forma, invadem predominantemente ecossistemas abertos (ex: savana, campo) e ambientes perturbados (ex: pasto abandonado), onde a disponibilidade de luz não é limitante (Martin et al., 2009). Logo, a restauração florestal, plantios florestais e sistemas agroflorestais são práticas de uso da terra que podem prevenir a invasão de espécies exóticas pelo estabelecimento de um filtro ambiental, como o sombreamento, que seleciona as espécies vegetais regenerantes por meio de suas preferências e características ecofisiológicas (Ramos et al., 2014).

Todos os exemplos documentados da presença de $A$. cunninghamiana como invasora são em sub-bosque de fragmentos florestais ou florestas restauradas maduras, nos quais essa palmeira exótica coexiste com outras espécies nativas tolerantes ao sombreamento (Dislich et al., 2002; Miranda Neto et al., 2012). Apesar do número de espécies exóticas tolerantes ao sombreamento ser relativamente menor que o de heliófitas, o impacto daquelas espécies pode ser ainda maior, pois ameaça os ambientes mais bem preservados e de maior valor de conservação (Martin et al., 2009).

Portanto, para remediar os problemas advindos da presença dessa palmeira invasora recomenda-se o corte de todos os indivíduos de palmeiras exóticas e enriquecimento por semeadura direta e ou plantio de mudas de Euterpe edulis (palmito-juçara), palmeira nativa que ocupa o mesmo nicho e é atrativa à fauna; enriquecimento através do plantio de mudas de espécies arbóreas típicas de estágios avançados de Floresta
Estacional Semidecidual da região; substituição gradual das palmeiras exóticas por Euterpe edulis, por meio do plantio intercalado de mudas desta espécie; retirada gradual de espécies não nativas da Floresta Estacional Semidecidual.

\section{Conclusões}

O estrato regenerante do povoamento de Araucaria angustifolia apresenta elevada densidade, mas diversidade e riqueza inferiores ao encontrado em Floresta Estacional Semidecidual madura na região de Viçosa, MG. Ademais, o sub-bosque estudado é composto, principalmente, por espécies arbustivas, tolerantes ao sombreamento, zoocóricas e por poucas regenerantes arbóreas. Merece destaque a espécie Archantophoenix cunninghamiana, palmeira exótica muito utilizada na ornamentação do campus da UFV, em Viçosa, que apresenta o maior valor de dominância da amostragem.

A. cunninghamiana foi caracterizada como uma invasora, que parece estar retardando o processo de sucessão secundária na área de estudo, tornando-se ameaça ao equilíbrio do ecossistema e à conservação da biodiversidade local. Entretanto, as normas criadas e ações de manejo definidas para a maioria das espécies exóticas não se aplica para aquelas que invadem o sub-bosque de ecossistemas florestais, como é o caso da palmeira australiana. Portanto, para áreas onde essa palmeira esteja presente com características de invasora, recomenda-se implementar técnicas para remediar o problema, visando não apenas sua erradicação, mas também a revegetação com espécies típicas de subbosque da região.

\section{Agradecimentos}

Os autores agradecem ao CNPq, pelas bolsas de iniciação científica concedida ao primeiro autor e de produtividade em pesquisa ao terceiro autor. Agradecem também à FAPEMIG, pela bolsa de doutorado concedida à segunda autora, e aos engenheiros florestais Isaac Jorge e Breno Arrivabeni pelo auxílio nos trabalhos de campo.

\section{Referências}

Alvares, C. A. et al. Köppen's climate classification map for Brazil. Meteorologische Zeitschrift, v. 22, n. 6, p. 711-728, 2013. DOI: 10.1127/0941-2948/2013/0507. 
The Angiosperm Phylogeny Group. An update of the Angiosperm Phylogeny Group classification for the orders and families of flowering plants: APG IV. Botanical Journal of the Linnean Society, v. 181, n. 1, p. 1-20, 2016. DOI: 10.1111/boj.12385.

Beckert, S. M. et al. Crescimento e dinâmica de Araucaria angustifolia (Bert.) O. Ktze. em fragmento de Floresta Ombrófila Mista. Scientia Forestalis, v. 42, n. 102, p. 209-218, 2014.

Callegaro, R. M. et al. Potencial de três plantações florestais homogêneas como facilitadoras da regeneração natural de espécies arbustivo-arbóreas. Scientia Forestalis, v. 41, n. 99, p. 331-341, 2013.

Camargos, V. L. et al. Avaliação do impacto do fogo no estrato de regeneração em um trecho de Floresta Estacional Semidecidual em Viçosa, MG. Revista Árvore, v. 34, n. 6, p. 1055-1063, 2010. DOI: 10.1590/S0100-67622010000600011.

Carvalho, P. E. R. Espécies florestais brasileiras: recomendações silviculturais, potencialidades e uso da madeira. Colombo: EMBRAPA-CNPF; Brasília, DF: EMBRAPA-SPI, 1994. 639 p.

Cavalheiro, C. N. et al. Distribuição de Piper gaudichaudianum Kuntze (Piperaceae) e efeito de borda em fragmento florestal do Jardim Botânico de Lajedo (JBL), Rio Grande do Sul. Revista Destaques Acadêmicos, v. 5, n. 3, p. 131-139, 2013.

Christianini, A. V. Fecundidade, dispersão e predação de sementes de Archontophoenix cunninghamiana $\mathrm{H}$. Wendl. \& Drude, uma palmeira invasora da Mata Atlântica. Revista Brasileira de Botânica, v. 29, n. 4, p. 587-594, 2006. DOI: 10.1590/S0100-84042006000400008.

Connell, J. H. \& Slatyer, R. O. Mechanisms of succession in natural communities and their role in community stability and organization. The American Naturalist, v. 111, p. 1119-1144, 1977.

Denslow, J. S. \& Dewalt, S. J. Exotic plant invasions in tropical forests: patterns and hypotheses. In: Carson, W. P. \& Schnitzer, S. A. (Ed.). Tropical forest community ecology. Chichester: WileyBlackwell, 2008. p. 409-426.

Dislich, R. et al. A invasão de um fragmento florestal em São Paulo (SP) pela palmeira australiana Archontophoenix cunninghamiana H. Wendl. \& Drude. Revista Brasileira de Botânica, v. 25, n. 1, p. 55-64, 2002. DOI: 10.1590/S0100-84042002000100008.

Durigan, G. \& Engel, V. L. Restauração de ecossistemas no Brasil: onde estamos e para onde podemos ir? In: Martins, S. V. (Ed.). Restauração ecológica de ecossistemas degradados. Viçosa, MG: Ed. da UFV, 2012. p. 41-68.

Eisenlohr, P. V. et al. Flora fanerogâmica do campus da Universidade Federal de Viçosa, Viçosa, Minas Gerais. Ceres, v. 55, n. 4, p. $317-$ 326, 2008.

Ferreira-Junior, W. G. et al. Influence of soils and topographic gradients on tree species distribution in a Brazilian Atlantic Tropical Semideciduous Forest. Edinburgh Journal of Botany, v. 64, n. 2, p. 137-157, 2007.

Font Quer, P. \& Sierra Ràfols, E. Diccionario de botánica. Barcelona: Labor, 1979. 1280 p.

Gandolfi, S. et al. Levantamento florístico e caráter sucessional das espécies arbustivo-arbóreas de uma floresta mesófila semidecídua no município de Guarulhos, SP. Revista Brasileira de Biologia, v. 55, n. 4, p. 753-767, 1995.
IBGE. Manual técnico da vegetação brasileira. 2. ed. Rio de Janeiro, 2012. 275 p.

Isernhagen, I. et al. Diagnóstico ambiental das áreas a serem restauradas visando a definição de metodologias de restauração florestal. In: Rodrigues, R. R. et al. Pacto pela restauração da Mata Atlântica: referencial dos conceitos e ações de restauração florestal. São Paulo: LERF/ESALQ: Instituto BioAtlântica, 2009. p. 87-126.

Jordano, P. et al. Ligando frugivoria e dispersão de sementes à biologia da conservação. In: Rocha, C. F. D. da et al. (Ed.). Biologia da conservação: essências. São Carlos: RIMA, 2006. p. 411-436.

Longhi, S. J. et al. Fatores ecológicos determinantes na ocorrência de Araucaria angustifolia e Podocarpus lambertii, na Floresta Ombrófila Mista da FLONA de São Francisco de Paula, RS, Brasil. Ciência Rural, v. 40, n. 01, p. 57-63, 2010. DOI: 10.1590/S010384782009005000220 .

Magurran, A. E. Medindo a diversidade biológica. Curitiba: Ed. da UFPR, 2011. 262 p.

Marangon, L. C. et al. Regeneração natural em um fragmento de Floresta Estacional Semidecidual em Viçosa, Minas Gerais. Revista Árvore, v. 32, p. 183-191, 2008. DOI: 10.1590/S010067622008000100020 .

Martin, P. H. et al. Why forests appear resistant to exotic plant invasions: Intentional introductions, stand dynamics, and the role of shade tolerance. Frontiers in Ecology and the Environment, v. 7, n. 3, p. 142-149, 2009. DOI: 10.1890/070096.

Meira-Neto, J. A. A. \& Martins, F. R. Estrutura do sub-bosque herbáceo-arbustivo da mata da silvicultura, uma floresta estacional semidecidual no município de Viçosa-MG. Revista Árvore, v. 27, n. 4, p. 459-471, 2003.

Melo, A. C. G. et al. Atributos de espécies arbóreas e a facilitação da regeneração natural em plantio homogêneo de mata ciliar. Scientia Forestalis, v. 43, n. 106, p. 333-344, 2015.

Miranda Neto, A. et al. Estrato de regeneração natural de uma floresta restaurada com 40 anos. Pesquisa Florestal Brasileira, v. 32, n. 72, p. 409-420, 2012. DOI: 10.4336/2012.pfb.32.72.409.

Moro, F. M. B. \& Martins, F. R. Métodos de levantamento do componente arbóreo-arbustivo. In: Felfili, J. M. et al. (Ed.). Fitossociologia no Brasil: métodos e estudos de casos. Viçosa, MG: Ed. da UFV, 2011. p. 174-208.

Müller-Dombois, D. \& Ellemberg, H. Aims and methods of vegetation ecology. Nova York: John Wiley \& Sons, 1974. 547 p.

Oliveira-Filho, A. \& Fontes, M. Patterns of floristic differentiation among Atlantic Forests in Southeastern Brazil and the influence of climate. Biotropica, v. 32, n. 4b, p. 793-810, 2000. DOI: 10.1111/ j.1744-7429.2000.tb00619.x.

Onofre, F. F. et al. Regeneração natural de espécies da Mata Atlântica em sub-bosque de Eucalyptus saligna Smith. em uma antiga unidade de produção forestal no Parque das Neblinas, Bertioga, SP. Scientia Forestalis, v. 38, n. 85, p. 39-52, 2010.

Paiva, H. N. et al. Cultivo do eucalipto: implantação e manejo. Viçosa, MG: Aprenda Fácil, 2011. 353 p.

Parker, I. M. et al. Impact: toward a framework for understanding the ecological effects of invaders. Biological Invasions, v. 1, p. 3-19, 1999. 
Pielou, E. C. Ecological diversity. New York: Jonhon Willey, 1975. $165 \mathrm{p}$.

Pijl, L. van der. Principles of dispersal in higher plants. 3nd. ed. New York: Springer-Verlag, 1982. 161 p.

Ramos, N. C. et al. Environmental filtering of agroforestry systems reduces the risk of biological invasion. Agroforestry Systems, v. 89, n. 2, p. 279-289, 2014. DOI: 10.1007/s10457-014-9765-7.

Reflora. Lista de espécies da flora do Brasil. Disponível em: $<\mathrm{http}: / /$ floradobrasil.jbrj.gov.br/>. Acesso em: 1 abr. 2016.

Ribeiro, T. M. et al. Restauração florestal com Araucaria angustifolia (Bertol.) Kuntze no Parque Estadual de Campos do Jordão, SP: efeito do fogo na estrutura do componente arbustivo-arbóreo. Scientia Forestalis, v. 40, n. 94, p. 279-290, 2012.
Richardson, D. M. \& Wilgen, B. W. van. Invasive alien plants in South Africa: how well do we understand the ecological impacts? South African Journal of Science, v. 100, n. 1, p. 45-52, 2004.

Silva Júnior, W. M. et al. Regeneração natural de espécies arbustivoarbóreas em dois trechos de uma Floresta Estacional Semidecidual, Viçosa, MG. Scientia Forestalis, n. 66, p. 169-179, 2004.

Souza, P. B. et al. Florística e estrutura da vegetação arbustivoarbórea do sub-bosque de um povoamento de Eucalyptus grandis W. Hill ex Maiden em Viçosa, MG, Brasil. Revista Árvore, v. 31, n. 3, p. 533-543, 2007. DOI: 10.1590/S0100-67622007000300019.

Thompson, I. D. et al. An operational framework for defining and monitoring forest degradation. Ecology and Society, v. 18, n. 2, 2013. DOI: 10.5751/ES-05443-180220.

Wilcove, D. S. et al. Quantifying threats to imperiled species in the United States. BioScience, v. 48, n. 8, p. 607-615, 1998. DOI: 10.2307/1313420. 